\title{
Expression of CXCR4 and CXCL12 and their correlations to the cell proliferation and angiogenesis in mycosis fungoides
}

\author{
Joanna Maj', Alina M. Jankowska-Konsur ${ }^{1}$, Agnieszka Hałoń², Zdzisław Woźniak², Ewa Plomer-Niezgoda ${ }^{1}$, Adam Reich ${ }^{1}$
}

1Department of Dermatology, Venereology and Allergology, Wroclaw Medical University, Wroclaw, Poland Head of the Department: Prof. Jacek Szepietowski MD, PhD

2Department of Pathomorphology, Wroclaw Medical University, Wroclaw, Poland

Head of the Department: Prof. Piotr Ziółkowski MD, PhD

Postep Derm Alergol 2015; XXXII (6): 437-442 DOI: $10.5114 /$ pdia.2015.48034

\begin{abstract}
Introduction: Chemokines play an important role in tumor growth, invasion and metastasis. The CXCR4/CXCL12 axis has been implicated in development of both solid tumors and hematological malignancies and is also relevant in the pathogenesis of the most common primary cutaneous T-cell lymphoma, mycosis fungoides (MF).

Aim: To evaluate the expression of CXCR4 and CXCL12 in MF and to examine their associations with cell proliferation and angiogenesis.

Material and methods: The material for the study consisted of skin samples obtained from 56 patients with MF and 20 healthy volunteers. The expression of CXCR4 and CXCL12 was assessed by immunohistochemistry on the paraffin blocks and compared to the expression of angiogenesis marker (CD34) and proliferation indicators (Ki-67, AgNORs). Results: The expression of chemokine CXCL12 and its receptor CXCR4 was significantly higher in MF than in the healthy skin $(p<0.001)$. There was no significant difference between early and advanced stages of MF. Similarly, there was no statistically important correlation between the expression of CXCR4/CXCL12 and angiogenesis and proliferation markers, however a significant correlation between CD34 and AgNORs expression was found $(p<0.001)$. Conclusions: The CXCR4/CXCL12 axis seems to play an important role in MF development in the early as well as in the advanced stages of the disease. Therefore, the CXCR4/CXCL12 axis seems to be an interesting potential target for the future strategies of new drug development, giving hope for more efficacious therapies for mycosis fungoides.
\end{abstract}

Key words: mycosis fungoides, CTCL, CXCR4, CXCL12.

\section{Introduction}

Mycosis fungoides (MF) represents the most common type of primary cutaneous lymphomas deriving from mature CD4-positive T cells (CTCL). Clinically, MF is characterized by indolent course and restriction to the skin, showing a slow evolution from macules and plaques to tumors. However, in some patients a rapid progression with metastases to lymph nodes and internal organs can be seen. Over the last decades, MF etiopathogenesis has been widely explored in depth, but still the exact mechanisms of initialization and development of this lymphoma have not been elucidated [1].

Chemokines are a superfamily of small, 8- to $12-\mathrm{kDa}$ peptides that function as chemoattractant cytokines. They mediate a number of cellular functions including activation, survival, growth, differentiation, and trafficking and exert their action by binding to surface, 7-transmembrane domain, G-protein coupled receptors on the target cells.

CXCL12 (C-X-C motif ligand 12), earlier termed stromal-derived factor-1 (SDF-1) is an $\alpha$-chemokine expressed on multiple cell types, including endothelial and stromal cells, in different organs. Its receptor, CXCR4, has been documented to be expressed on most hematopoietic cell types, including CD34+ progenitor cells, CD4+ T cells, B cells, neutrophils and dendritic cells. Additionally, the expression of CXCR4 has been found on multiple solid tumors as well as hematological malignancies. Growing evidence has accumulated that the CXCL12/CXCR4 axis plays an important role in multiple

Address for correspondence: Alina M. Jankowska-Konsur MD, PhD, Department of Dermatology, Venereology and Allergology, Wroclaw Medical University, 1 Chałubińskiego St, 50-368 Wroclaw, Poland, phone: +48 691033 632, e-mail: alina.jankowska-konsur@am.wroc.pl Received: 24.03.2014, accepted: 8.07.2014. 
processes such as stem cell mobilization, migration and homing, inflammation, infection and immunoregulation. The mechanisms employing the CXCR4/CXCL12 axis have been also implicated in tumor development, growth and metastasis [2].

\section{Aim}

The aim of the study was to analyze the expression pattern and intensity level of CXCR4 and CXCL12 in the primary cutaneous lymphoma tissues and to compare with the expression of well-known markers of tumor progression (AgNORs, Ki-67, CD34).

\section{Material and methods}

The material for the immunohistochemical analysis consisted of 56 archival tissue samples from the patients with histologically-proven mycosis fungoides fixed in $10 \%$ buffered formalin solution and embedded in paraffin blocks. The diagnosis and staging of the disease was assessed according to the WHO/EORTC classification and revised ISCL/EORTC classification and staging $[3,4]$. The control group consisted of 20 healthy volunteers surgically treated in our department for cosmetic reasons.

\section{The immuno-pathomorphological analysis of CXCR4 and CXCL12 expression}

The $5 \mu \mathrm{m}$ thick slices were prepared from the paraffin blocks containing fixed tissue, then dewaxed in xylene and placed on basic slides Super Frost Plus (Menzel $\mathrm{GmbH} \&$ Co KG, Germany). Separate slides for the assessment of the tested markers CXCR4 and CXCL12 were performed from each block. Following commercial primary antibodies against studied proteins were used for the immunohistochemical staining:

- CXCR4: Monoclonal Anti-human CXCR4 (fusin) Antibody; Clone: 44716; Ig class: IgG2B; Catalog Number: MAB 172; R\&D SYSTEMS; dilution 1 : 120;

- CXCL12: Monoclonal Anti-human/mouse CXCL12/SDF-1 Antibody; Clone: 79018; Ig class; mouse IgG1; Catalog Number: MAB 350; R\&D SYSTEMS USA; dilution $1: 60$.

The immunohistochemical procedure was performed as follows: Before the incubation with the primary antibody the antigens were exposed by boiling and incuba- tion in $0.01 \mathrm{M}$ sodium citrate buffer ( $\mathrm{pH}$ 6.0). The activity of endogenous peroxidase was blocked with a blocking agent (Peroxidase-blocking Reagent, S2001, DAKO, Denmark) and rinsed in distilled water and phosphatebuffered saline (PBS) solution. Nonspecific immunoglobulin binding sites were blocked with the normal serum diluted with PBS, $0.1 \%$ bovine serum albumin (BSA) and $0.1 \%$ sodium azide. Then, samples were incubated with the primary antibody (standard dilution, recommended by the manufacturer with Antibody Diluent, Background Reducing (S3022, DAKO, Denmark)). Next, the biotinylated antibody was added to the samples, which were incubated at room temperature for 20 min and again rinsed in PBS. Streptavidin-biotinylated peroxidase complex (Universal LSAB ${ }^{\oplus}$ Kits HRP, Rabbit/Mouse/Goat, (K 0690, DAKO, Denmark)) was added, incubated and rinsed. After staining with DAB, samples were rinsed in distilled water. Nuclei were stained with hematoxylin. Finally, the slides were dehydrated with alcoholic series and xylene and closed in DPX. Positive and negative controls were done for every staining procedure. The results of immunohistochemical staining was analyzed on the basis of the system of immunocytochemical reaction assessment using an IRS immunocytochemistry (Immunoreactive score) by Remmel own modification (Table 1) [5].

According to this scale two expression categories are evaluated separately: 1) percentage of cells showing a positive staining - from 0 to 4 points (\% - percentage) (Table 1), 2) degree of color reaction - from 0 to 3 points ( 1 - intensity of the reaction).

The final scoring is a result of multiplication of $\%$ and I (from 0 to 12 points) and is defined as the ratio of IRS - RIRS.

Additionally, topography of the protein expression in lymphoma infiltration was evaluated as two categories: 1) superficial - the dominant expression pattern in the superficial infiltrate (within the epidermis or close to the epidermis), 2) deep - the dominant expression in the deep infiltrate (in the deeper layers of the skin).

\section{Immunohistochemical analysis of CD34, AgNORs, and $\mathrm{Ki}-67$ expression}

The following primary antibodies were used for the detection of CD34 and Ki-67: Monoclonal Mouse Antihuman CD34, clone: Class II, QBEnd 10 (DAKO, Catalog

Table 1. IRS Assessment scale according to Remmel (own modification) [5]

\begin{tabular}{lccc}
\hline Percentage of cells showing reaction (\%) & Points & Intensity of reaction (I) & No reaction \\
\hline Lack of the cells exhibiting reaction & 0 & Weak color reaction & 0 \\
\hline $1-25 \%$ of positive cells & 1 & Moderate color reaction & 2 \\
\hline $26-50 \%$ of positive cells & 2 & Intense color reaction \\
\hline $51-75 \%$ of positive cells & 3 &
\end{tabular}


Table 2. Expression of CXCL12 and CXCR4 in mycosis fungoides (MF) and healthy skin (IRS - immunoreactive score)

\begin{tabular}{|c|c|c|c|c|}
\hline \multirow[t]{2}{*}{ Variable } & \multicolumn{2}{|c|}{ CXCL12 } & \multicolumn{2}{|c|}{ CXCR4 } \\
\hline & MF & Healthy skin & MF & Healthy skin \\
\hline \multicolumn{5}{|c|}{ Percentage of positive cells: } \\
\hline $0-10 \%(1)$ & $2(3.6 \%)$ & $12(60.0 \%)$ & 4 (7.1\%) & $4(20.0 \%)$ \\
\hline $11-50 \%(2)$ & $27(48.2 \%)$ & $7(35.0 \%)$ & $16(28.6 \%)$ & $15(75.0 \%)$ \\
\hline $51-80 \%$ (3) & $21(37.5 \%)$ & $0(0 \%)$ & 21 (37.5\%) & $1(5 \%)$ \\
\hline \multirow[t]{2}{*}{$>80 \%(4)$} & $6(10.7 \%)$ & $1(5.0 \%)$ & $15(26.8 \%)$ & $0(0 \%)$ \\
\hline & \multicolumn{2}{|c|}{$<0.001$} & \multicolumn{2}{|c|}{$<0.001$} \\
\hline \multicolumn{5}{|l|}{ Staining intensity: } \\
\hline Low (1) & $2(3.6 \%)$ & $13(65 \%)$ & $2(3.6 \%)$ & $16(80 \%)$ \\
\hline Intermediate (2) & $29(51.8 \%)$ & $7(35 \%)$ & $30(53.6 \%)$ & $4(20 \%)$ \\
\hline \multirow[t]{2}{*}{ High (3) } & 25 (44.6\%) & $0(0 \%)$ & $24(42.9 \%)$ & $0(0 \%)$ \\
\hline & \multicolumn{2}{|c|}{$<0.001$} & \multicolumn{2}{|c|}{$<0.001$} \\
\hline \multirow[t]{2}{*}{ IRS, mean \pm SD } & $6.3 \pm 2.8$ & $1.4 \pm 0.8$ & $7.1 \pm 3.2$ & $2.2 \pm 1.0$ \\
\hline & \multicolumn{2}{|c|}{$<0.001$} & \multicolumn{2}{|c|}{$<0.001$} \\
\hline \multicolumn{5}{|l|}{ Staining location: } \\
\hline Superficial & 23 (41.1\%) & $6(30 \%)$ & 25 (44.6\%) & $16(80 \%)$ \\
\hline Deep & 5 (8.9\%) & $0(0 \%)$ & 5 (8.9\%) & $0(0 \%)$ \\
\hline Both & $28(50 \%)$ & $1(5 \%)$ & $26(46.4 \%)$ & $4(20 \%)$ \\
\hline \multirow[t]{2}{*}{ None } & $0(0)$ & $13(65 \%)$ & - & - \\
\hline & \multicolumn{2}{|c|}{$<0.001$} & \multicolumn{2}{|c|}{0.02} \\
\hline
\end{tabular}

Number N1632) and Monoclonal Mouse Anti-human Ki-67, clone: MIB-1, (DAKO catalog number N1633) were used. The exact staining procedure was described in detail elsewhere [6, 7].

To visualize proteins NORs, the preparations were stained according to Ploton modified by Ofner [8]. The whole procedure was described previously [6].

\section{Statistical analysis}

Statistical analysis was performed using Statistica ${ }^{\oplus}$ 9.0 software (Statsoft, Krakow, Poland). Frequencies, mean, median, minimal and maximal values were calculated, where appropriate. Students $t$ test, Mann-Whitney $U$ test, $\chi^{2}$ test and Spearman rank correlation test were used for statistical analysis. Values of $p$ less than 0.05 were considered significant.

\section{Results}

\section{Expression of CXCL12 and CXCR4}

The comparison of the CXCL12 and CXCR4 expression in biopsies of MF and healthy skin is demonstrated in Table 2. Both, CXCL12 and CXCR4 were expressed in more cells of lymphoma specimens than in cells of the control skin (Figure 1). Similarly, lesional skin of MF showed significantly higher immunoreactivity of CXCL12 and CXCR4 than healthy skin. The immunoreactivity score (IRS) for CXCL12 and CXCR4 was significantly higher ( $p<0.001$, $p<0.001)$ in mycosis fungoides $(6.3 \pm 2.8$ scores and 7.1 \pm 3.2 scores, respectively) than in control biopsies ( $1.4 \pm 0.8$ scores and $2.2 \pm 1.0$ scores, respectively) (Table 2).

\section{Relevance of CXCL12 and CXCR4 in MF}

To establish the importance of CXCL12 and CXCR4 for the prognosis of mycosis fungoides we have compared them with the well-known markers of tumor progression like Ki67, CD34 and AgNORs (Figures 2, 3). We did not observe any significant correlations between IRS of CXCL12 and CXCR4 and the expression of analyzed proliferation and angiogenesis markers (Table 3). On the other hand, the expression of all three proliferation parameters significantly correlated between themselves (Table 3). The age and gender of included patients did not significantly influence the expression of any of the studied parameters (data not shown).

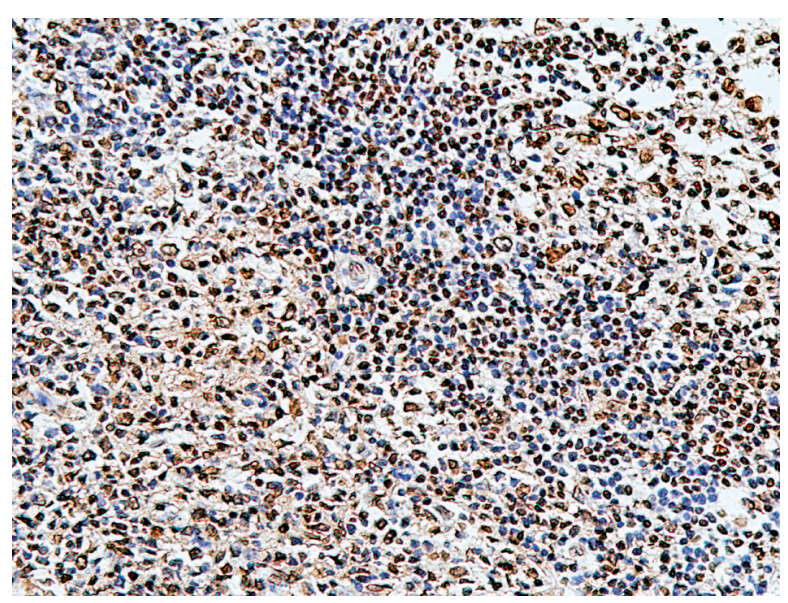

Figure 1. The expression of CXCR4 in mycosis fungoides (magnification 200x) 


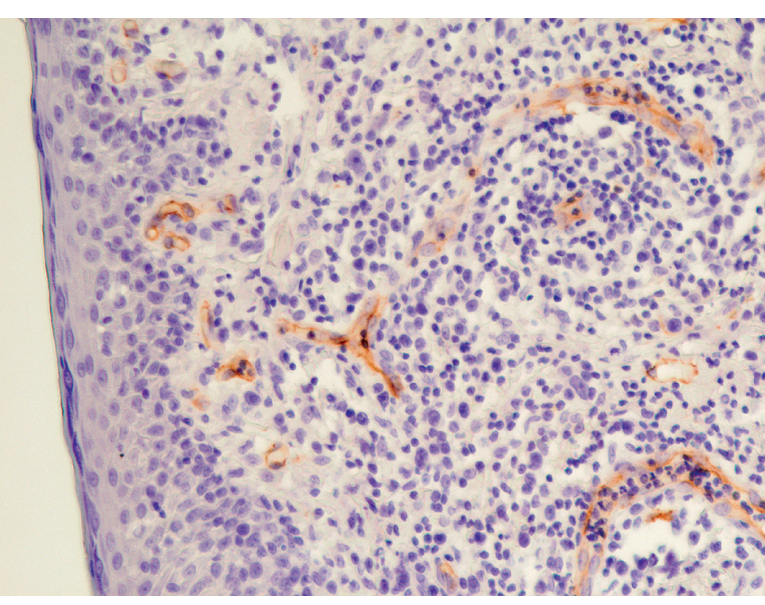

Figure 2. The expression of CD34 in mycosis fungoides tissue (magnification 200x)

When comparing the expression of Ki67, CD34, AgNORs, CXCL12 and CXCR4 with the disease status we observed that parameters of cell proliferation and angiogenesis had a significantly higher expression in more advanced stages of MF and were connected with shorter survival, whereas the expression of CXCL12 and CXCR4 did not significantly correlate with the disease course (Table 4). We also did not find any significant relationships between the localization of CXCL12 and CXCR4 staining in the skin and the disease stage and patient status (Table 4).

\section{Discussion}

The expression of CXCR4 has been observed in various tumor types, including solid tumors as well as hematological malignancies. This receptor has been shown to be employed in various processes such as chemotaxis, invasion, angiogenesis and proliferation.

It has been postulated that the CXCR4/CXCL12 axis plays an important role in regulation of metastasis processes, similarly to the physiological processes of the retention/homing of hematopoietic stem cells into the bone marrow microenvironment, by trafficking CXCR4 positive malignant cells to the tissues secreting CXCL12

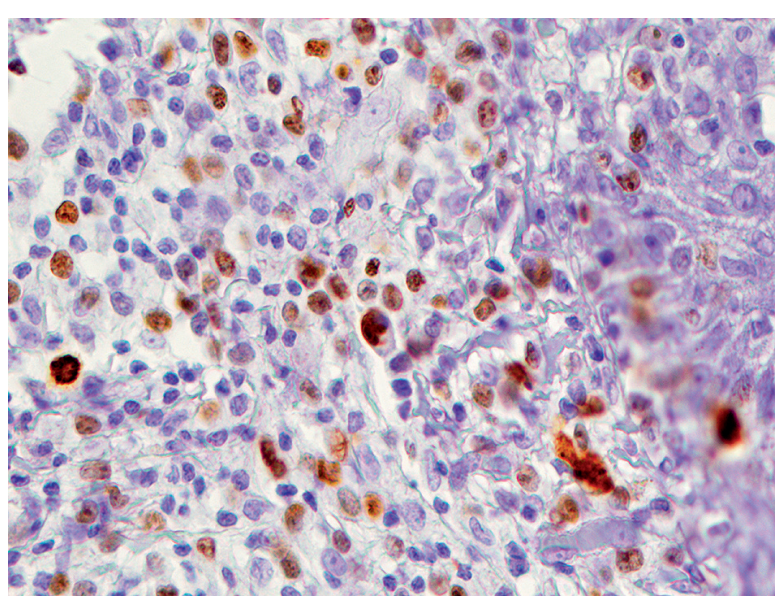

Figure 3. The expression of $\mathrm{Ki}-67$ in mycosis fungoides (magnification 400x)

[9]. The mechanism of CXCR4-CXCL12-induced metastases to the bone marrow was suggested in lung cancer [10]. The high CXCR4 expression on melanoma malignum cells was reported by other authors who found a strong correlation between CXCR4 expression and unfavorable prognosis and shorter median disease-free and overall survival [11]. The other reports documented the association between the overexpression of CXCR4 on malignant cells with poor overall survival in patients with breast and ovarian cancer $[12,13]$. Similar processes were also reported in hematological malignancies. In acute lymphoblastic leukemia (ALL) it has been documented in vivo that CXCR4 expression plays a key role in the engraftment of ALL cells in the bone marrow microenvironment [14]. Moreover, CXCR4 expression and its interaction with adhesion molecules are necessary for the homing of malignant cells to the bone marrow and their resistance to cytotoxic therapies in acute myelogenous leukemia and it has been found to be a negative prognostic factor [15, 16]. It has been postulated that in B- and T-cell non-Hodgkin lymphomas, the expression of CXCR4 on the malignant cells plays a role in lymphoma cell trafficking and homing. Skin homing of malignant lymphocytes is a hallmark of MF and Sezary syndrome (SS - aggressive variant of (TCL) [17]. This process is based on an interaction

Table 3. Correlations between the expression of CXCL12, CXCR4 and proliferation markers ( $\rho$ - Spearman correlation coefficient) in MF

\begin{tabular}{lccccc}
\hline & Ki67 & MVD & AgNORs & CXCL12 IRS & CXCR4 IRS \\
\hline Ki67 & X & $\rho=0.68, p<0.001$ & $\rho=0.79, p<0.001$ & $\rho=0.25, p=0.06$ & $\rho=0.05, p=0.72$ \\
\hline CD34 & $\rho=0.68, p<0.001$ & $X$ & $\rho=0.8, p<0.001$ & $\rho=0.18, p=0.2$ & $\rho=-0.02, p=0.9$ \\
\hline AgNORs & $\rho=0.79, p<0.001$ & $\rho=0.8, p<0.001$ & $X$ & $\rho=0.23, p=0.1$ & $\rho=0.04, p=0.75$ \\
\hline CXCL12 IRS & $\rho=0.25, p=0.06$ & $\rho=0.18, p=0.2$ & $\rho=0.23, p=0.1$ & $\mathrm{X}$ & $\rho=-0.04, p=0.76$ \\
\hline CXCR4 IRS & $\rho=0.05, p=0.72$ & $\rho=-0.02, p=0.9$ & $\rho=0.04, p=0.75$ & $\rho=-0.04, p=0.76$ & $\mathrm{X}$ \\
\hline
\end{tabular}


Table 4. Comparison of the expression of Ki67, CD34, AgNORs, CXCL12, CXCR4 with the disease course and prognosis

\begin{tabular}{|c|c|c|c|c|c|}
\hline Variable & $\begin{array}{c}\text { Ki67 } \\
\text { (\% of cells) }\end{array}$ & $\begin{array}{c}\text { CD34 } \\
\text { (microvessel } \\
\text { density } / \mathrm{mm}^{3} \text { ) }\end{array}$ & $\begin{array}{l}\text { AgNORs } \\
\text { (number) }\end{array}$ & $\begin{array}{l}\text { CXCL12 IRS } \\
\text { (scores) }\end{array}$ & $\begin{array}{c}\text { CXCR4 IRS } \\
\text { (scores) }\end{array}$ \\
\hline \multicolumn{6}{|l|}{ MF stage: } \\
\hline Early $(I A-I \mid A)$ & $9.4 \pm 9.8$ & $121.6 \pm 11.4$ & $1.7 \pm 0.7$ & $5.1 \pm 1.8$ & $7.3 \pm 3.2$ \\
\hline \multirow{2}{*}{ Advanced (IIB-IVB) } & $20.5 \pm 10.3$ & $138.2 \pm 20.9$ & $3.6 \pm 1.2$ & $6.7 \pm 2.9$ & $7.0 \pm 3.3$ \\
\hline & $p<0.001$ & $p<0.01$ & $p<0.001$ & $p=0.07$ & $p=0.81$ \\
\hline \multicolumn{6}{|l|}{ Metastases: } \\
\hline No & $13.9 \pm 8.2$ & $126.9 \pm 16.9$ & $2.6 \pm 1.1$ & $6.0 \pm 2.7$ & $7.2 \pm 3.2$ \\
\hline \multirow{2}{*}{ Yes } & $27.4 \pm 12.3$ & $151.7 \pm 17.1$ & $4.3 \pm 1.2$ & $6.9 \pm 3.0$ & $6.9 \pm 3.5$ \\
\hline & $p<0.001$ & $p<0.001$ & $p<0.001$ & $p=0.32$ & $p=0.74$ \\
\hline \multicolumn{6}{|l|}{ Patient status: } \\
\hline Alive & $16.4 \pm 11.4$ & $130.7 \pm 19.4$ & $2.9 \pm 1.3$ & $4.7 \pm 3.3$ & $5.6 \pm 3.6$ \\
\hline \multirow{2}{*}{ Dead } & $22.9 \pm 9.4$ & $146.2 \pm 19.3$ & $4.0 \pm 1.3$ & $6.5 \pm 2.2$ & $6.9 \pm 3.2$ \\
\hline & $p=0.07$ & $p=0.02$ & $p=0.01$ & $p=0.08$ & $p=0.74$ \\
\hline
\end{tabular}

between CXCR4 expressed on lymphocytes and CXCL12 expressed on the fibroblasts and stromal cells in the dermis and may be enhanced by another characteristic feature of MF/SS, namely, loss of the CD26 surface antigen. Physiologically, CD26 cleaves and inactivates CXCL12 and its down-regulation increases CXCL12-dependent chemotaxis of SS cell lines [18]. The CXCL12 expression in the tumor microenvironment provides stimulatory signals for B-cells and activated T cells in chronic leukemia [19]. Similar phenomena may also occur in CTCL. High expression and immunoreactivity of CXCR4 and CXCL12 found by our group in MF further supports an important role of this axis in the development of this malignancy.

Uncontrolled cell proliferation is a characteristic feature of the tumor growth. Previously, we assessed the cell proliferation rate by measuring Ki-67 and AgNORs expression in MF and parapsoriasis en plaque and we found an increased expression of these biomarkers in advanced MF as compared to early MF and parapsoriasis [6]. Taking into consideration numerous reports suggesting that the CXCR4/CXCL12 axis is involved in tumor growth promotion, in the current study we have compared the expression of proliferation markers with the expression of CXCR4 and CXCL12. Interestingly, the expression of CXCR4 and CXCL12 did not correlate with tumor progression biomarkers expression as well as did not influence the patient outcome suggesting separate mechanisms employed in both processes.

There is also growing evidence of the association between the CXCR4/CXCL12 axis and angiogenesis in tumor development by the up-regulation of the VEGF and VEGFR, one of the most potent proangiogenic factors and its receptor [20]. Several reports linked the increased microvessel density (MVD) with a high expression of CXCR4 and CXCL12 and tumor progression in myelodysplastic syndrome and lung cancer [21-23]. Increased angiogenesis measured by MVD in cutaneous lymphomas was reported by our group elsewhere [7], however, in view of the presented data we compared MVD measured by CD34 staining to the CXCR4/CXCL12 expression. Both MVD and CXCR4/CXCL12 expressions were increased in the lymphoma group, but there was no correlation between them, which again may suggest different mechanisms of both processes: skin homing and angiogenesis. Recently, another receptor - CXCR7, which also binds CXCL12, was found to stimulate angiogenesis in vitro and in vivo in the rat mammary adenocarcinoma cell line MTLn3, whereas CXCR4 enhanced tumor invasiveness [24].

\section{Conclusions}

There is growing evidence that chemokines and their receptors play an important role in pathogenesis of MF. Our study seems to confirm by the close relationship between the expression of CXCR4/CXCL12 axis and the MF development. Several anti-CXCL12 and CXCR4 agents, including the anti-CXCR4 drug AMD3100 (plerixafor), the CXCL12 analog CTCE-9908, the anti-CXCL12 aptamer Nox-A12, have been tested with promising effects in preclinical and clinical studies as an adjunctive antitumor therapy. The CXCR4/CXCL12 axis seems to be an interesting potential target for future strategies of new drug development, giving hope for more efficacious therapies for solid tumors and hematological malignancies, including MF.

\section{Conflict of interest}

The authors declare no conflict of interest.

\section{References}

1. Sokołowska-Wojdyło M, Jankowska-Konsur A, Grzanka A. Patogeneza ziarniniaka grzybiastego i zespołu Sezary'ego. Przegl Dermatol 2012, 99: 235-40.

2. Burger JA, Kipps TJ. CXCR4: a key receptor in the crosstalk between tumor cells and their microenvironment. Blood 2006; 107: 1761-7. 
3. Willemze R, Jaffe ES, Burg G, et al. WHO/EORTC classification for cutaneous lymphomas. Blood 2005; 105: 3768-85.

4. Olsen E, Vonderheid E, Pimpinelli N, et al. Revisions to the staging and classification of mycosis fungoides and Sezary syndrome: a proposal of the International Society for Cutaneous Lymphomas (ISCL) and the cutaneous lymphoma task force of the European Organization of Research and Treatment of Cancer (EORTC). Blood 2007; 110: 1713-22.

5. Remmel W, Schicketanz KH. Immunohistochemical determination of estrogen and progesterone receptor content in human breast cabcer. Computer-assisted image analysis (QIC score) vs. subjective grading (IRS). Pathol Res Pract 1993; 189: 862-6.

6. Maj J, Woźniak Z, Białynicki-Birula R, Jankowska-Konsur A. Ocena markerów proliferacji w ziarniniaku grzybiastym i przyłuszczycy plackowatej. Dermatol Klin 2008; 10: 207-10.

7. Jankowska-Konsur A, Maj J, Woźniak Z, Baran E. Ocena angiogenezy w ziarniniaku grzybiastym. Postep Derm Alergol 2009; 26: 186-9.

8. Ofner D, Bankfakvi A, Riehemann K, et al. Wet autoclave pretreatment improves the visualization of silver-stained nucleolar organizer-region-associated proteins in routinely formalin fixed and paraffin embedded tissues. Mod Pathol 1994; 7: 946-50.

9. Ma Q, Jones D, Springer TA. The chemokine receptor CXCR4 is required for the retention of $B$ lineage and granulocytic precursors within the bone marrow microenvironment. Immunity 1999; 10: 463-71.

10. Phillips RJ, Burdick MD, Lutz M, et al. The stromal derived factor-1/CXCL12-CXC chemokine receptor 4 biological axis in non-small cell lung cancer metastases. Am J Respir Crit Care Med 2003; 167: 1676-86.

11. Scala S, Ottaiano A, Ascierto PA, et al. Expression of CXCR4 predicts poor prognosis in patients with malignant melanoma. Clin Cancer Research 2005; 11: 1835-41.

12. Li YM, Pan Y, Wei Y, et al. Upregulation of CXCR4 is essential for HER2-mediated tumor metastasis. Cancer Cell 2004; 6 : 459-69.

13. Guo L, Cui ZM, Zhanq J, Huanq Y. Chemokine axes CXCL12/ CXCR4 and CXCL16/CXCR6 correlate with lymph node metastasis in epithelial ovarian carcinoma. Chin J Cancer 2011; 30: 336-43.

14. Sipkins DA, Wei $X$, Wu JW, et al. In vivo imaging of specialized bone marrow endothelial microdomains for tumour engraftment. Nature 2005; 435: 969-73.

15. Mohle R, Bautz F, Rafii S, et al. The chemokine receptor CXCR4 is expressed on CD34+ hematopoietic progenitors and leukemic cells and mediates transendothelial migration induced by stromal cell-derived factor-1. Blood 1998; 91: 4523-30.

16. Rombouts EJ, Pavic B, Lowenberg B, et al. Relation between CXCR-4 expression, Fit3 mutations and unfavorable prognosis of adult acute myeloid leukemia. Blood 2004; 104: 550-7.

17. Wu X, Lonsdorf AS, Hwang ST. Cutaneous T cell lymphoma: roles for chemokines and chemokines receptors. J Invest Dermatol 2009; 129: 1115-9.

18. Narducci MG, Scala E, Bresin A, et al. Skin homing of Sezary cells involves SDF-1 -CXCR4 signaling and down-regulation of CD26/dipeptidylpeptidase IV. Blood 2006; 107: 1108-5.

19. Borge M, Nannini PR, Morande PE, et al. CXCL12 is a costimulator for CD4(+) T cell activation and proliferation in chronic lymphocytic leukemia patients. Cancer Immunol Immunother 2013; 62: 113-24.
20. Ping YF, Yao XH, Jiang JY, et al. The chemokine CXCL12 and its receptor CXCR4 promote glioma stem cell-mediated VEGF production and tumour angiogenesis via PI3K/AKT signalling. J Pathol 2011; 224: 344-54.

21. Zhang $\mathrm{Y}$, Zhao $\mathrm{H}$, Zhao D, et al. SDF-1/CXCR4 axis in myelodysplastic syndromes: correlation with angiogenesis and apoptosis. 2012; 36: 281-6.

22. Franco R, Pirozzi G, Scala S, et al. CXCL12-binding receptors expression in non-small cell lung cancer relates to tumoral microvascular density and CXCR4 positive circulating tumoral cells in lung draining venous blood. Eur J Cardiothorac Surg 2012; 41: 368-75.

23. Cui K, Zhao W, Wang C, et al. The CXCR4-CXCL12 pathway facilitates the progression of pancreatic cancer via induction of angiogenesis and lymphangiogenesis. J Surg Res 2011; 171: 143-50.

24. Hernandez L, Magalhaes MA, Coniglio SJ, et al. Opposing roles of CXCR4 and CXCR7 in breast cancer metastasis. Breast Cancer Res 2011; 13: R128. 